\title{
Array-based carbon black-polymer composite vapor detectors for detection of DNT in environments containing complex analyte mixtures
}

Shawn M. Briglin, Michael S. Freund, Brian C. Sisk, Nathan Saul Lewis

Shawn M. Briglin, Michael S. Freund, Brian C. Sisk, Nathan Saul Lewis, "Array-based carbon black-polymer composite vapor detectors for detection of DNT in environments containing complex analyte mixtures," Proc. SPIE 4394, Detection and Remediation Technologies for Mines and Minelike Targets VI, (18 October 2001); doi: 10.1117/12.445419

SPIE Event: Aerospace/Defense Sensing, Simulation, and Controls, 2001, Orlando, FL, United States 


\title{
Array Based Carbon Black-Polymer Composite Vapor Detectors for Detection of DNT in Environments Containing Complex Analyte Mixtures
}

\author{
Shawn M. Briglin, Michael S. Freund, Brian C. Sisk, Nathan S. Lewis ${ }^{\mathrm{a}}$, \\ ${ }^{a}$ Division of Chemistry and Chemical Engineering, Noyes Laboratory, 127-72 \\ California Institute of Technology, Pasadena, CA 91125
}

\begin{abstract}
Thin films of carbon black-organic polymer composites have been deposited across two metallic leads, with sorption of vapors producing swelling-induced resistance changes of the detector films. To identify and classify vapors, arrays of such vapor sensing elements have been constructed in which each element of the array contains a different polymer as the insulating phase and a common conductor, carbon black, as the conducting phase. The differing gas-solid partition coefficients for the various polymers of the detector array produce a pattern of differential resistance changes that is used to classify vapors and vapor mixtures. The performance of this detector array system towards 2,4-dinitrotoluene, the predominant signature in the vapor phase above land mines, in the presence high concentrations of water or of acetone (as a selected volatile organic carbon vapor), has been evaluated.
\end{abstract}

Keywords: Land mine signatures, vapor detectors, 2,4-dinitrotoluene, carbon black

\section{INTRODUCTION}

Prior work in our laboratory has utilized broadly responsive detector arrays based on carbon black composites ${ }^{1-3}$ for various vapor detection tasks. In this approach, films consisting of carbon black dispersed into different insulating organic polymers form the individual detector elements. The carbon black endows electrical conductivity to the films, whereas the different organic polymers provide the source of chemical diversity between elements in the detector array. Swelling of polymers $^{4-11}$ upon exposure to a vapor increases the resistance of the film, thereby providing a remarkably simple means for monitoring the presence of a vapor. ${ }^{1-3}$ Because different polymer compositions are present in each detector element, an array of elements responds to a wide variety of vapors (or binary mixtures of vapors) in a distinctive, identifiable fashion. The electrical resistance signals that comprise the output from the array can be readily integrated into software or hardware-based decision systems, allowing for an integration of sensing and analysis functions into a compact, low-power, device.

In the present work, we have focused on the performance of such carbon black-polymer composite detector arrays towards 2,4-dinitrotoluene (DNT). This compound has been reported to comprise the predominant vapor phase signature above land mines, ${ }^{12}$ and the presence of DNT has been associated with the success of canines as well as with the success of novel vapor detection schemes for locating buried mines in the field. ${ }^{13}$ As with any scheme that utilizes arrays of broadly responsive detectors to detect and identify an analyte at extremely low concentrations, the presence of high concentrations of one or more interfering vapors may cause errors in target detection or recognition. We describe herein the implementation of a simple technique capable of removing one or more of these background vapors to yield the signal pattern of the desired target species even when the background vapor is present at concentrations in excess of $10^{5}-10^{6}$ higher than that of the target compound.

In addition we address the question of the performance of a broadly-responsive detector array as a function of the number of chemically distinct materials used to form vapor detectors. A significant issue in the use of arrays of semiselective vapor detectors is how the performance of the system depends on the number of chemically distinct detectors in the array. Hughes et. al. have claimed that four conducting polymer composite detectors are sufficient to provide a "universal solvent detector." 14 Park et. al. found that for certain analyte classification tasks, arrays of 3-6 detectors provided comparable results, and these authors concluded that increasing the number of detectors in an array did not improve performance significantly. ${ }^{15}$ In contrast, measurements using conducting polymer composite detectors have indicated that the performance in certain vapor classification tasks improved as the number of different detectors in a conducting polymer composite array increased. ${ }^{1}$ The relationship between the number of different detectors and overall system performance is important because 
significant engineering tradeoffs are faced for surface acoustic wave (SAW) devices, quartz crystal microbalances, conducting polymer composites, and dye-impregnated optical beads or optical fibers as the number of detectors is increased. For example, more SAW detectors require increased power consumption; increased numbers of optical detectors require higher pixel counts in the detector; and increased numbers of chemiresistors require more rapid analog-to-digital converters to maintain the same overall system data acquisition frequency. Increasing the number of detectors in an array also requires more computational power to preprocess, process, and analyze the resulting data stream. The focus of the work reported herein is to address in a quantitative fashion how the performance of differently sized arrays of conducting polymer composite vapor detectors performs for certain vapor detection tasks.

To compare quantitatively the relative performance of various detector arrays, the array must be presented with pairs of analytes that will not be perfectly classified by at least some of the arrays. This was not the case with pairs of simple organic vapors presented at relatively high concentration, all of which were essentially perfectly separated from each other, including structural isomers such as ortho- and meta-xylene. ${ }^{3}$ As part of this work, we have challenged a carbon black polymer composite detector array with mixtures of a pair of compounds that are very chemically similar, hexane and heptane. The responses of the carbon black composite detectors are linear with analyte concentration and the response of a binary mixture of analytes is the response of the pure analytes weighted by the mole fraction of analytes in the mixture. ${ }^{2}$ Thus, the Euclidean distance between a binary vapor mixture that is 0.5 mole fraction of each constituent and a binary mixture that is a 0.6:0.4 mixture of these same analytes should be one tenth of the Euclidean distance between the array responses of the individual pure analytes. These analyte mixtures therefore present relatively challenging discrimination tasks for a broadly responsive vapor detector array.

If a broadly responsive array-based vapor detection system were blindly exposed to n-hexane/n-heptane mixtures, an intermediate level task likely would involve determination of the components of the mixture. However, first-level tasks would likely involve establishing that the analytes in the mixtures were hydrocarbons and perhaps that the analytes had a chain length of approximately 5-6 carbons. The next level task would be to identify the hydrocarbons as hexane and heptane, and final level task would be to determine the relative concentrations of each component of the mixture. Obviously, the first step in the process is the determination of the class, or functional group, of the analyte to which the detector array was exposed. This process, however, is made more difficult by the fact that many molecules are members of multiple classes. An example would be fluorobenzene, which is both a halogen and an aromatic. Accordingly, methods for class determination are explored herein using the best five detectors in a selection of two-class separation tasks.

\section{EXPERIMENTAL}

Two separate types of experiments were performed. The procedures for each set of experiments are described briefly in this section.

\subsection{Response at Constant Flow Rate of a Detector Array in the Presence of Volatile Organic Compounds and Water.}

Saturated DNT vapor at $21^{\circ} \mathrm{C}$ was obtained from a glass tube approximately one meter in length that held $\approx 180 \mathrm{~g}$ of loosely packed, granulated DNT. The air flow through this tube was $200 \mathrm{ml} \mathrm{min}^{-1}$, with the background gas being oil-free laboratory air $(1.10 \pm 0.15$ parts per thousand (ppth) of water vapor). An additional gas stream passed through a bubbler that contained either acetone or water. Two in-line union-T's were used to mix the DNT vapor stream, the stream that contained either of the "interfering" vapors, and a background laboratory air gas stream. Flows were controlled with Teflon solenoid valves and mass flow controllers, in a computer-controlled system which has been described in detail previously. ${ }^{2}$ A short Teflon tube was connected to the output of the union to direct the gas toward the bank of detectors. The total flow rate of the gas directed at the detectors was held constant at $2 \mathrm{~L} \mathrm{~min}^{-1}$ during all parts of the experiment. The DNT concentration after dilution was $10 \%$ of its vapor pressure. At this dilution, the upper limit of the DNT concentration is 14 parts per billion (ppb) because the vapor pressure of DNT at room temperature is approximately $140 \mathrm{ppb}$. When present in the vapor stream, the concentration of the acetone was 12.9 parts per thousand (ppth). Although the background air stream always contained some water vapor, the concentration was roughly doubled to $\approx 2.3$ ppth during exposures that contained water as an "interfering" vapor. During exposures of the detector array, the vapor stream contained either pure DNT, water, or acetone; mixtures of DNT and water vapor; or mixtures of DNT and acetone vapor. Analyte exposures were $10 \mathrm{~min}$ in duration, and were separated in time by a 40 min exposure to the background air stream.

Nine detector composite types were used, each fabricated from a different insulating polymeric phase. The following materials were used to form these insulating phases for the detectors of the corresponding number: 
Table 1 - List of Detector Materials

Detector Material:

1. PEVA (25\% VA)

2 Polyethylene oxide

3. Polycaprolactone

4. Poly(vinyl stearate)

5. Polyvinylacetate + diethylene glycol dibenzoate $50 \%(\mathrm{wt} / \mathrm{wt})$
Detector Material:

6. PMMA + diethylene glycol dibenzoate $50 \%(\mathrm{wt} / \mathrm{wt})$

7. PEVA (45\% VA)

8. Styrene/isoprene

9. polymethyloctadecyl siloxane

The composites used in this experiment were sprayed onto three circuit board substrates as illustrated in Figure 1.

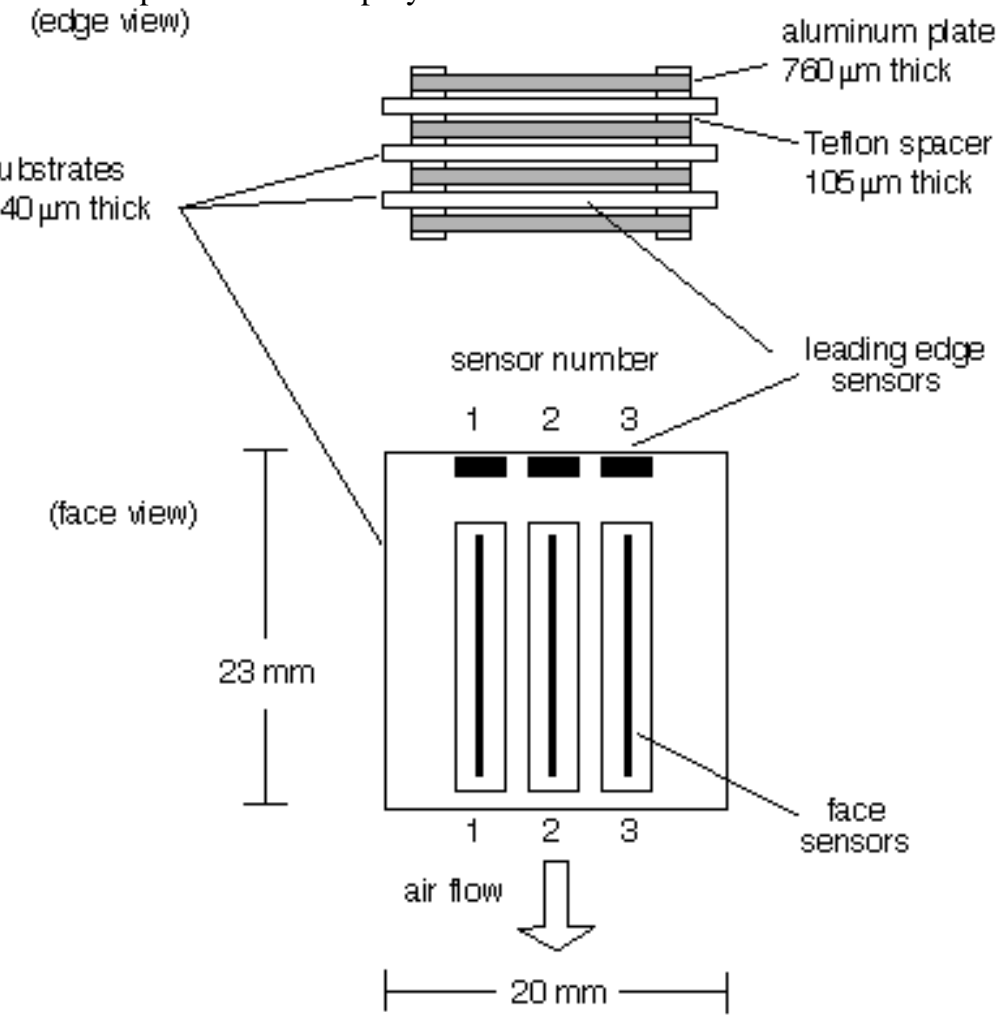

Figure 1: Circuit board substrates used to assemble the high-low vapor pressure optimized stacking detector assembly. Each face detector was prepared with the same polymer composite as was used in the edge detector directly in front of the face detector, and three face detectors were used. This created nine detector film types in each of the two positions, making a total of eighteen detectors.

Each of these substrates had electrical contacts deposited in a pattern that created a total of six detectors. Three detectors were located on the face of the substrate and three on the edge of the substrate. The three leading edge detectors were formed on the $840 \mu \mathrm{m}$ thick edge of the substrate between parallel contacts that were located on each face of the circuit board. These detectors were located in positions 1,2 and 3 of Figure 1. The $20 \mathrm{~mm}$ by $23 \mathrm{~mm}$ faces of the circuit board supported the three larger detectors, each of which had dimensions of $2.0 \mathrm{~mm}$ by $15 \mathrm{~mm}$. The electrodes that formed face detectors in the same location on the top and bottom of each substrate were wired together in parallel (i.e. the leads to face detector 1 on the top face were connected in parallel to the leads that addressed face detector 1 on the bottom face of the substrate). On each substrate this arrangement therefore produced three face detectors, each having a total film area of $60 \mathrm{~mm}^{2}(2 \times 2.0 \mathrm{~mm} \times 15$ $\mathrm{mm})$. Three of these substrates were stacked so that their leading edges were normal to the flow, and the flow through the gaps was controlled with a pump at $100 \mathrm{ml} \mathrm{min}^{-1}$; consequently, the total flow of the diluted vapor stream between each chip was much lower than that directed at the edge detectors. 


\subsection{Vapor Delivery System and Detector Array Setup for Assessment of Discrimination Performance}

The vapor delivery system utilized in the second set of experiments consisted of up to eight solvent bubblers capable of delivering mixtures of solvents to a detector array containing as many as 40 detectors. The resistance of the detectors was measured as a function of time, as described previously. ${ }^{3}$ The baseline resistance, $R_{b}$, of each detector was calculated from the average of the resistance readings for the $60 \mathrm{~s}$ immediately prior to the beginning of the exposure. The equilibrium response, $R_{\text {eq }}$, was calculated from the average of the resistance readings for the last $60 \mathrm{~s}$ of the exposure. The quantity used in data analysis was the steady-state relative differential resistance change, $\Delta R / R_{b}$, where $\Delta R=R_{e q}-R_{b}$. To correct for drift, the baseline used was determined by a linear fit from the first ten data points of the pre-exposure period. For the experiments described herein, each mixture or solvent was presented to the array 10 times, and data are averages over these replicate exposures. Analytes were exposed to the detectors in random order. Each exposure protocol consisted of $180 \mathrm{~s}$ exposure to background air, $180 \mathrm{~s}$ to the analyte of interest, and finally another $420 \mathrm{~s}$ of exposure to background air.

The following detectors were used:

Table 2 - List of Detector Materials

\begin{tabular}{llll} 
Detector Material: & Solvent: & Detector Material: & Solvent: \\
\hline 1. Polyethylene oxide & toluene & 11. polycaprolactone & THF \\
2. PEO-PAMAM \#1 & chloroform & 12. p-chloroprene & THF \\
3. PEVA (45\% VA) & toluene & 13. polysulfone & THF \\
4. PEO-PAMAM \#1 & chloroform & 14. PANI-0.5-HDBSA & HFIP \\
5. Poly(styrene-b-butadiene) & toluene & 15. polyvinylpyrrolidone & ethanol \\
6. Kraton G1152 & toluene & 16. bis (cyanoallyl polysiloxane) & THF \\
7. Polyvinylcarbazole & THF & 17. poly-4-vinylphenol & THF \\
8. Kraton G1108 & toluene & 18. poly(styrene-allyl alcohol) & toluene \\
9. Polyvinylacetate & acetone & 19. polymethyloctadecyl siloxane & THF \\
10. p-diphenoxyphosphazene & THF & 20. ethyl hydroxyethyl cellulose & toluene
\end{tabular}

\subsection{Linear Discriminant Analysis:}

Fisher's linear discriminant is an algorithm that maximizes the variance between groups, while minimizing the variance within groups, with the groups being user-defined. When down-selecting the best five detectors for a particular task, linear discriminant analysis was run exhaustively for each combination of five out of 20 total detectors.

\subsection{Discrimination Between Mixtures of N-hexane and N-heptane}

11 mixtures of hexane and heptane were created by mixing bubbler streams from two bubblers. The first bubbler generated a hexane stream at a fraction A multiplied by $0.02 \mathrm{P}_{\text {hexane }}^{\mathrm{o}}$, where $\mathrm{P}_{\text {hexane }}^{\mathrm{o}}$ is the partial pressure of hexane at room temperature and pressure. The second bubbler produced a heptane stream at a fraction B multiplied by $0.02 \mathrm{P}_{\text {heptane. For all }}^{\mathrm{o}}$ mixtures, $\mathrm{A}+\mathrm{B}=1$. These mixtures are shown below:

Table 3 - N-hexane/n-heptane mixtures use

\begin{tabular}{ccccc} 
Mixture & Hexane Multiplier & Hexane, $\mathrm{P} / \mathrm{P}^{\mathrm{o}}$ & Heptane Multiplier & Heptane $\mathrm{P} / \mathrm{P}^{\mathrm{o}}$ \\
\hline 1. & 1.00 & 0.0200 & 0.00 & 0.0000 \\
2. & 0.90 & 0.0180 & 0.10 & 0.0020 \\
3. & 0.80 & 0.0160 & 0.20 & 0.0040 \\
4. & 0.70 & 0.0140 & 0.30 & 0.0060 \\
5. & 0.60 & 0.0120 & 0.40 & 0.0080 \\
6. & 0.50 & 0.0100 & 0.50 & 0.0100 \\
7. & 0.40 & 0.0080 & 0.60 & 0.0120 \\
8. & 0.30 & 0.0060 & 0.70 & 0.0140 \\
9. & 0.20 & 0.0040 & 0.80 & 0.0160 \\
10. & 0.10 & 0.0020 & 0.90 & 0.0180 \\
1. & 0.00 & 0.0000 & 1.00 & 0.0200
\end{tabular}




\subsection{Discrimination Between Solvent Classes:}

The following pure solvents were used:

Table 4 - Solvents Analyzed

\begin{tabular}{lllll} 
Alcohols & Halogens & Aromatics & Hydrocarbons & Esters \\
\hline 2-butanol & 1-bromobutane & benzene & n-hexane & isopropyl acetate \\
1-pentanol & 1-bromopentane & propyl benzene & n-octane & butyl acetate \\
2-pentanol & 3-chloro-2-methyl-propene & m-xylene & n-decane & isobutyl acetate \\
3-pentanol & 1-chloropropane & o-xylene & n-heptane & hexyl acetate \\
isopropanol & 2-chlorobutane & p-xylene & n-nonane & pentyl acetate \\
ethanol & 1-iodopropane & isopropyl benzene & n-pentane & methyl acetate \\
1-butanol & 2-bromo-2-methylpropane & ethyl benzene & cyclohexane & propyl acetate \\
2-methyl-1-propanol & 1-iodobutane & toluene & cyclopentane & sec-butyl acetate \\
3-methyl-1-butanol & chloroform & 1-fluorobenzene & cyclooctane & isoamyl acetate \\
3-methyl-2-butanol & methylene chloride & 1-chlorobenzene & & ethyl propionate \\
2-propen-1-ol & 1,1,2-trichloroethylene & & & isoamyl propionate \\
1-hexanol & 1-chlorobenzene & & & amyl butrate \\
2-methyl-3-butene-2-ol & 1-fluorobenzene & & & ethyl butyrate \\
& & & & 2,6 lutidene
\end{tabular}

Solvents were used as supplied from commercial sources with no further purification.

\section{RESULTS}

\subsection{Response to 10 minute Pulses of DNT Vapor at Constant Flow Rate}

Figure 2 shows the $\Delta \mathrm{R} / \mathrm{R}$ response of the array of 18 detectors to DNT and to mixtures of DNT that contained high concentrations of either acetone or water vapor.

\section{Raw Responses to Pure DNT Vapor Dilutions and DNT Vapor Dilutions Containing High Concentrations of Contaminant Vapors}

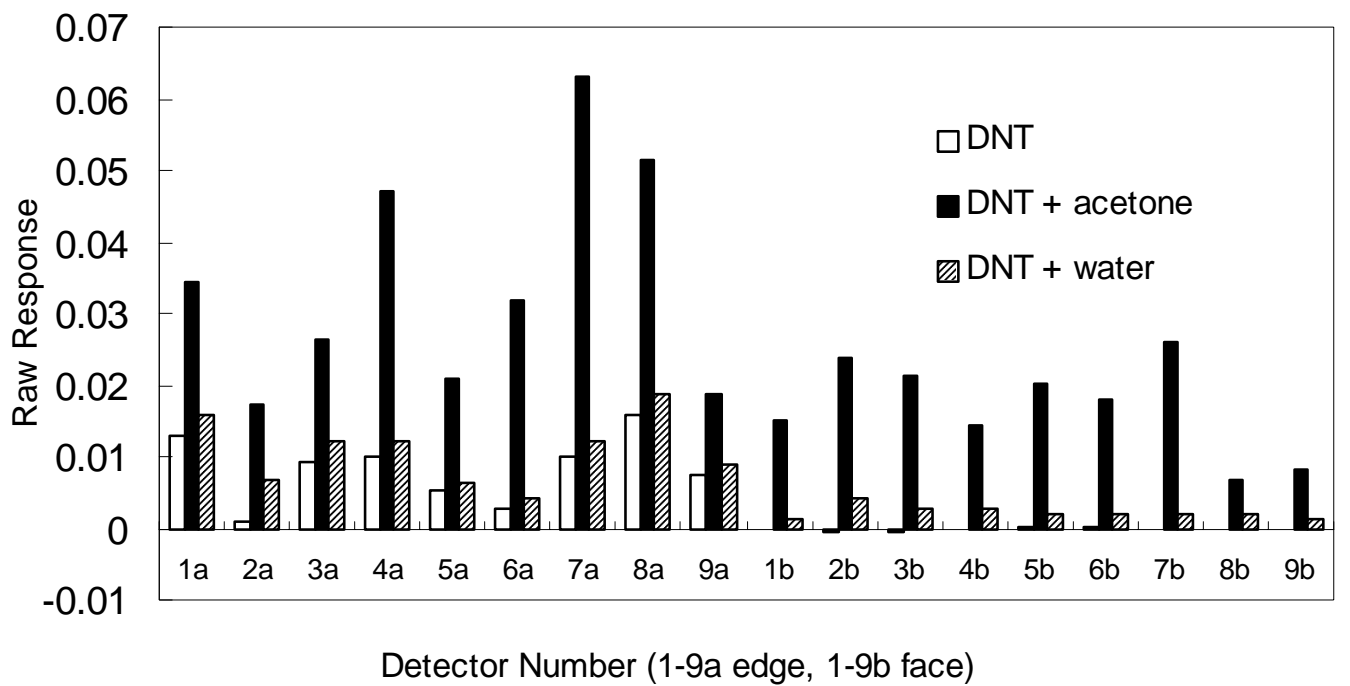

Figure 2: Average $\Delta \mathrm{R} / \mathrm{R}$ response computed as the baseline normalized differential resistance change of the detectors for 10 presentations of each vapor or mixture after 10 minute exposures to ppb levels of DNT in the presence of ppth levels of two potentially interfering compounds. 
For pure analytes, vapors with small polymer/gas partition coefficients (generally analytes with high vapor pressures) produced similar magnitude signals on the leading edge and the corresponding face detector having the same composite material. In contrast, virtually all of the DNT (having a low vapor pressure and therefore a high polymer/gas partition coefficient in general) was trapped on the leading edge detectors and produced essentially no response on the face detectors. For mixtures that contained both DNT and high vapor pressure analytes, subtraction of the face detector response from the edge detector response yielded the response of only the low vapor pressure (high polymer/gas partition coefficient) component of the vapor mixture. Because the responses of carbon black-polymer composite films are well known to be linear with respect to concentration and additive with respect to components of binary mixtures, ${ }^{2}$ subtraction techniques of this type can be applied without prior knowledge of the concentration or response pattern of the interfering vapor or knowledge of the effectiveness of the mass transport of the DNT vapor to the detector film.

The responses to the high vapor pressure analyte on the large face detectors were first corrected by the slight variation in the relative sensitivity to both types of individual detectors (face and edge) and then subtracted to yield the response pattern of the pure DNT. This variation in sensitivity is expected to be independent of the concentration of the interfering analyte, permitting this correction to made against unknown concentrations of any contaminant analyte exhibiting small polymer/gas partition coefficients.

\section{Extrapolated DNT Pattern in the Presence of High Concentrations of Contaminant Vapors}

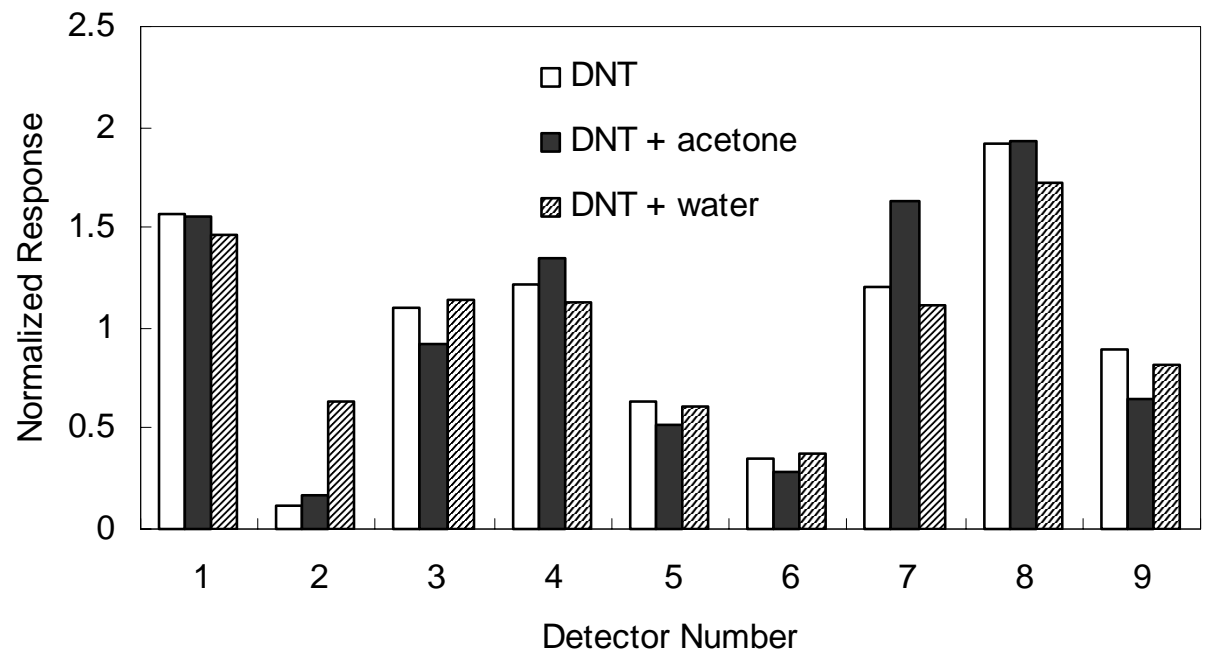

Figure 3: Normalized array fingerprint patterns of pure DNT, and DNT in the presence of large concentrations of acetone or water.

As can be seen in Figure 3, the extrapolated response pattern of the detectors is very similar to that of pure DNT even though the DNT was in the presence of much higher concentrations of acetone or water. Although the pre-equilibrium (time dependent) response pattern of the detectors to DNT or to any other analyte with a very high partition coefficient is expected to depend more closely on the film thickness of the individual detectors than on the specific interactions between the analyte and polymers of the individual detectors, the response pattern of the detectors to DNT is expected to be characteristic and is therefore useful in elucidating the existence of such a compound in the presence of high concentrations of interfering low partition coefficient compounds. Because responses of carbon black-polymer composite are additive in nature, ${ }^{2}$ subtraction techniques of this type could potentially remove an unlimited number of unknown interfering VOC's and water present simultaneously from the array pattern of DNT, provided that the relative sensitivity to these analytes on face and edge detectors is similar, as expected, for a given polymer composite. This hardware-based preprocessing capability clearly circumvents many of the limitations of software-based pattern matching algorithms based on the face detector response alone, which would require prior knowledge of the array response to the specific interfering analyte and would encounter difficulties with the occurrence of high numbers of vapors simultaneously present in the vapor surrounding the DNT target. 


\subsection{Detector Array Size Evaluation}

In the second set of experiments, we investigated the ability of detector arrays to discriminate between chemically similar analytes. For this purpose, the performance of subsets of detectors was compared to that of the full set of detectors for discrimination between mixtures of hexane and heptane. Five detectors were chosen that maximized the product of the resolution values for every separation. Resolution is defined in eq 1, where $\delta$ is the distance between the two population

$$
\text { resolution }=\frac{\delta}{\sqrt{\left(\sigma_{1}^{2}\right)+\left(\sigma_{2}^{2}\right)}}
$$

means and $\sigma_{1}$ and $\sigma_{2}$ are the two population standard deviations. For each pair of hexane/heptane mixtures, a set of five out of the 20 detectors was down-selected such that the resolution between the particular analyte pair (i.e. between one hexane/heptane mixture and another hexane/heptane mixture) was maximized. If a single set of five detectors were optimal for all tasks, then the same five detectors should be obtained repeatedly for all of these separation tasks by the down-selection procedure.

Figure 4 depicts maps showing which detectors were contained in down-selected optimally performing subsets for each particular analyte discrimination task. In these figures, the heptane content increased moving down and to the right on the graphs, with each element representing a single pairwise mixture separation task. No detectors were picked along the diagonal because all elements on the main diagonal correspond to the nonphysical task of a mixture being separated from itself. Elements near the diagonal, however, correspond to separations of hexane/heptane mixtures that are very compositionally similar to each other.

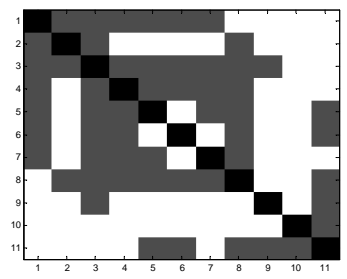

(a)

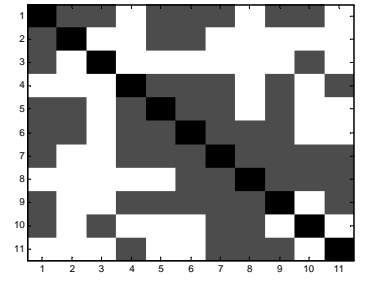

(b)

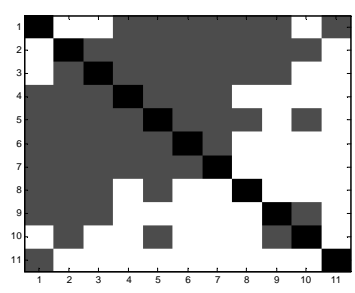

(c)

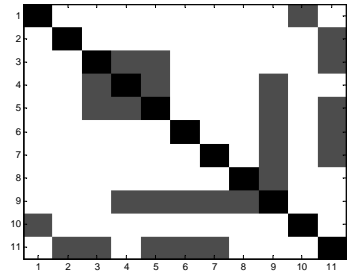

(d)

Figure 4: Distribution of analyte mixture separations for which these detectors were picked among the best five down-selected detectors for each separation task. The lighter shades represent separations for which each detector was contained in the down-selected detector set. Separations were not performed along the main diagonal. The detectors were carbon black composites formed from a) polyethylene oxide, b) PEVA, c) polycaprolactone and d) ethyl hydroxyethyl cellulose.

A number of the detectors showed significant spatial distributions in Figure 4, indicating that in some cases they formed members of an optimally performing subset of detectors whereas in other cases they were not included in the downselected optimally performing subset for the task of interest. Not all detectors exhibited a clear pattern to their selection. For example, ethyl hydroxyethyl cellulose was frequently contained in down-selected subsets without any clear pattern to the distribution.

The detectors that were down-selected for a given separation were not completely repeatable from run to run, as runspecific fluctuations affected which detectors were picked in the down-selected set for classification of that particular mixture data. To remove this run-to-run variability, each mixture was presented to the detectors a second time, with data for each pair of mixtures were separated using two sets of detectors. One set consisted of the detectors that were down-selected for that specific separation and data set, and the other set consisted of a subset of detectors that was selected as optimum based on all of the previous run's hexane/heptane mixture data. The detectors selected for a specific task generally outperformed the detectors that were chosen based on optimal performance from the whole data set. Using data from three different days of data collection, detector sets were chosen based on one day of data collection, and these detectors were used to perform classification tasks with data collected on the other two days. Two resolution values were thus determined for each 
separation task, and the ratio of these two values indicated how well the individual best five detectors compared to those down-selected for overall performance. Taking the log of these ratios and averaging over the 55 distinct separations possible for a given data set provided an indication of how well the two detector sets performed relative to each other for all of the mixtures, whereas using this average as the argument of a base-ten exponential returned the value to a usable ratio. In this case, the values obtained were $1.15,1.43 .1 .63,0.65,2.90$, and 0.80 . A value over 1 indicated that the detector sets obtained from analysis of individual exposure data outperformed the subsets that provided optimal performance for the entire set of data. This analysis showed that there was significant variation as to whether it was advantageous, under the conditions tested, to use a generalized group of five detectors or to use the set that worked best previously for a particular separation task. The advantage for the group set, when it occurred, likely arose from the fact that the detectors had been tested over a larger set of data, and would fluctuate less between runs.

\subsection{Class Separations}

Each analyte class consisted of between 10 and 16 members, as shown above in Table 4. Each analyte was presented 10 times to an array of carbon black-polymer composite vapor detectors and a full data set was collected when the class members were exposed in random order to the array. Linear discriminant analysis was then used to determine the resolution between each pair of analyte classes. As can be seen from the list above, some analytes are members of more than one class. These analytes were omitted from the assessment of interclass separability performance.

Resolutions between classes were found to be:

Table 5 - Resolutions Between Classes Using All 20 Detectors

\begin{tabular}{llllll} 
& Alcohols & Halogens & Aromatics & Hydrocarbons & Esters \\
\hline Alcohols & N/A & 10.7652 & 9.402 & 6.7424 & 6.5878 \\
Halogens & & N/A & 2.4455 & 5.8108 & 9.0768 \\
Aromatic & & N/A & 7.6891 & 5.2762 \\
Hydrocarbons & & & N/A & 9.7441 \\
Esters & & & & N/A
\end{tabular}

All of the classes were clearly well separated from each other, with the exception of the halogen/aromatic discrimination. Examples of linear discriminant separations, specifically hydrocarbons vs. both aromatics and halogens are displayed in Figure 5. Note that when either halogens or aromatics were compared to hydrocarbons, fluorobenzene was mapped onto the correct side of the decision boundary.

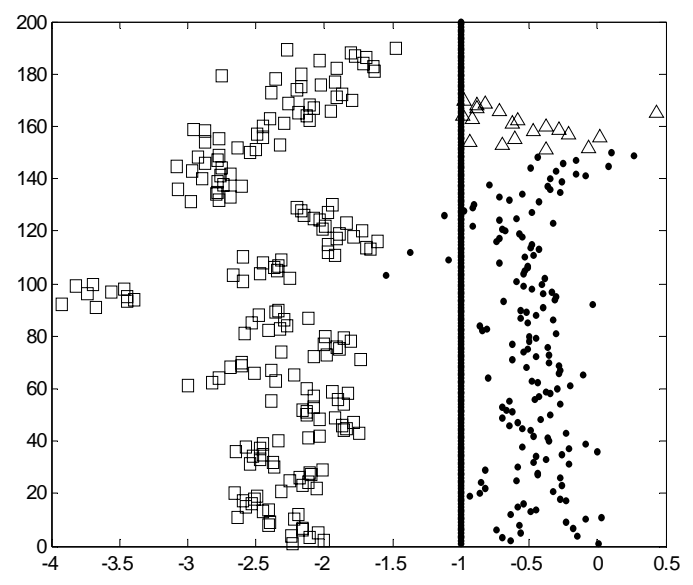

(a)

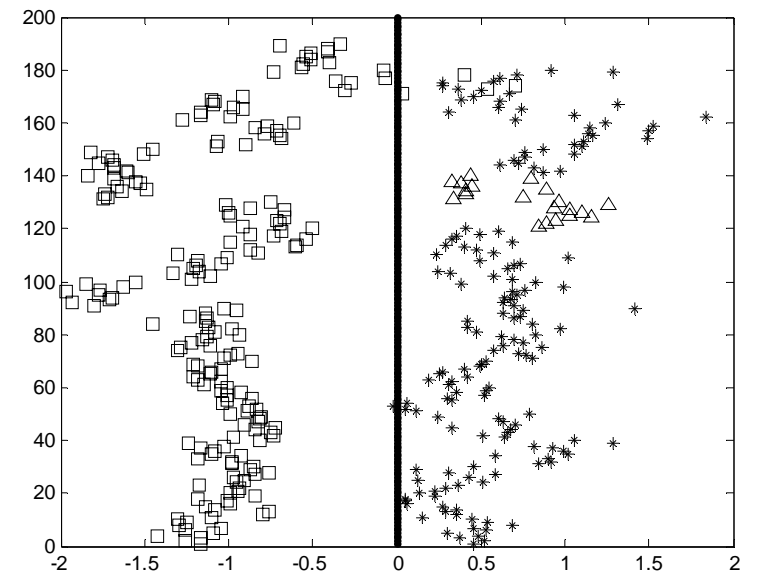

(b)

Figure 5: Linear Discriminant Separations of hydrocarbons from a) aromatics, and b) halogens. In a), sensors 1,2,3,11, and 15 were used to separate the two groups. Dots represent aromatics. In b), sensors 1,3,8,10, and 11 were used to separate the two groups. Asterisks represent halogens. In both plots, squares represent hydrocarbons, and the solid line represents the decision boundary, and triangles represent fluorobenzene, a member of both halogens and aromatics. 
If a single set of five detectors were chosen for separation of these data into analyte classes, even the best 5membered subset of detectors produced a significant reduction in the resolution factor between classes. The reduction in pairwise resolution factor was $18 \%$ on average and the reduction ranged from $2 \%$ to $47 \%$ for the various class separation tasks. For pairs of classes that were robustly differentiated with the full detector set, this degradation of resolution factor would not not be a significant problem in terms of incorrectly classing solvents into their correct classes. However, for other class separation tasks, significant classification error rates were introduced by such a down-selection procedure. Assuming that any points within one standard deviation of the decision boundary are unclassifiable, the number of such exposures in the halogens vs. aromatic class separation rose from $23 \%$ to $28 \%$ of the total when using a down-selected set of five detectors. This percentage of unclassifiable exposures increased to $45 \%$ of the total points when the best five down-selected detector subset was used for the same separation.

Table 6 - Resolutions between classes using individual and group "best five" arrays

\begin{tabular}{llclr} 
Classes Separated & Best 5 & Resolution(Ind. Best) & Resolution(Group Best) & Ratio \\
\hline Alcohols, Aromatics & $3,6,8,14,18$ & 8.3291 & 5.2970 & .6360 \\
Alcohols, Halogens & $1,3,6,8,10$ & 7.9898 & 6.7289 & .8422 \\
Alcohols, Hydrocarbons & $1,3,4,6,10$ & 5.6421 & 5.1407 & .9111 \\
Alcohols, Esters & $3,4,5,6,10$ & 5.3382 & 3.4921 & .6542 \\
Halogens, Aromatics & $6,7,8,11,18$ & 2.1699 & 1.9437 & .8958 \\
Halogens, Hydrocarbons & $1,3,8,10,11$ & 5.7329 & 5.2125 & .9092 \\
Halogens, Esters & $1,3,6,10,12$ & 5.1724 & 2.8105 & .5434 \\
Aromatics, Hydrocarbons & $1,2,3,11,15$ & 6.6251 & 6.2483 & .9431 \\
Aromatics, Esters & $1,2,3,11,15$ & 4.6432 & 4.0999 & .9431 \\
Hydrocarbons, Esters & $1,3,8,11,14$ & 8.7203 & 8.5668 & .9824
\end{tabular}

The data for the class separation task were recorded over a period of months, so run-peculiar and non-repeatable variations should be minimally incorporated into the weights and down-selected detector subsets. To evaluate this hypothesis, the detectors and weights found for the alcohol/hydrocarbon separation were applied to a large number of 1propanol/2-propanol mixtures, as well as to the hexane/heptane mixtures described above, which were run months later. As seen in Figure 6, the means of the data shifted significantly toward the decision boundary. However, the spread of the data was reasonable, suggesting that a relatively few number of exposures to a standard might be sufficient to re-center the means, as opposed to requiring a complete redetermination of the appropriate weight vectors. The extent to which trends in such data can be learned and such partial knowledge incorporated into updated training vectors is a focus of current work in our laboratory, however it appears to be a promising approach to extending the validity of the training set beyond an initially determined period of performance based on the initial training set data separation.

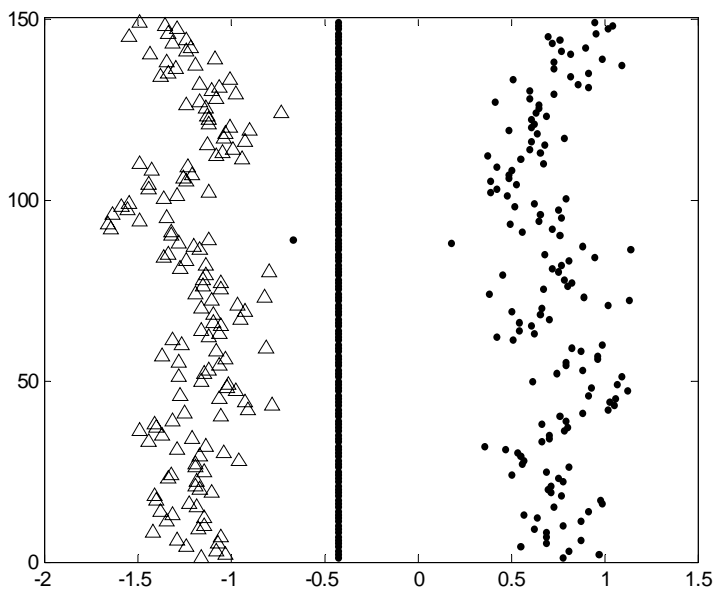

(a)

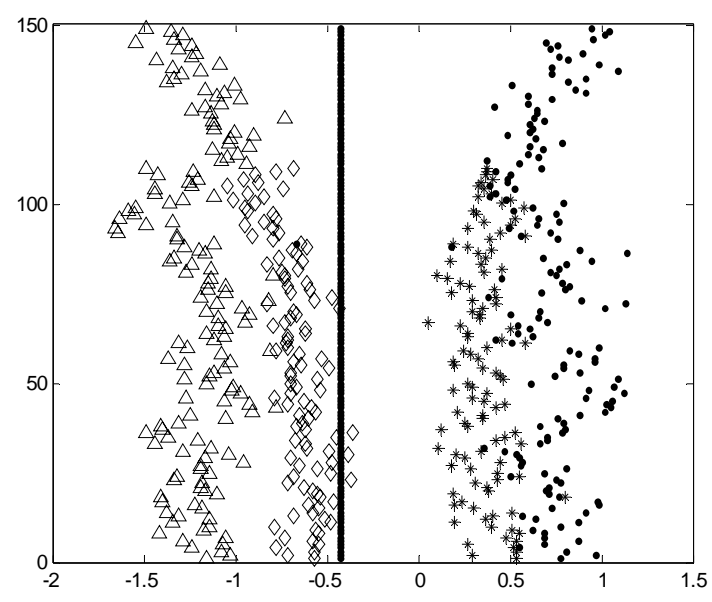

(b)

Figure 6: Separation of a) alcohols from hydrocarbons and b) the application of the mapping weight vector to sets of $n$-hexane/n-heptane mixtures and 1-propanol/2-propanol mixtures. The means shifted over time, but that the spread did not increase significantly. In both figures, dots correspond to alcohols and triangles correspond to hydrocarbons. In the second figure, asterisks correspond to alcohols, diamonds to hydrocarbons. 


\section{CONCLUSIONS}

In conclusion, we have shown that it is possible to use differential signal cancellation methods to minimize signals from unwanted, high vapor pressure interferences when detecting low vapor pressure analytes such as DNT vapors for use in detecting vapor-based land mine signatures. Alternatively, the method can be used to reject signals from unwanted low vapor (high polymer/gas partition coefficient) analytes in the presence of analytes to be detected that have higher vapor pressures. Additionally, we have shown that different subsets of detectors in an array provide optimal performance for different vapor classification tasks, and that such classification performance between analyte classes is relatively robust over significant

(months) time periods without retraining of the detector array.

\section{ACKNOWLEDGMENTS}

We acknowledge an OSD ARO MURI for demining, DOE, and an ARO olfactory research MURI for support of this work

\section{REFERENCES}

(1) Doleman, B. J.; Lonergan, M. C.; Severin, E. J.; Vaid, T. P.; Lewis, N. S. Anal. Chem. 1998, 70, 4177.

(2) Severin, E. J.; Doleman, B. J.; Lewis, N.S. Anal. Chem.; 2000; 72; 658

(3) Vaid, T. P.; Burl, M. C.; Lewis, N.S. Anal. Chem. 2001, 73, 321.

(4) Norman, R. H. Conductive Rubbers and Plastics; Elsevier: Amsterdam, 1970.

(5) Ford, C. J. In U.S. Patent 2,691,134, 1951.

(6) Newton, R. G. J. Rubber Res. 1946, 15, 35.

(7) Sands, A. G.; McDowell, M. V. Rubber Age, New York 1956, 80, 500.

(8) Boyd, J.; Bulgin, D. J. Text. Inst. Proc. 1957, 48, 66.

(9) Lundberg, B.; Sundqvist, B. J. Appl. Phys. 1986, 60, 1074.

(10) Ruschau, G. R.; Newnham, R. E.; Runt, J.; Smith, B. E. Sens. Actuators 1989, 20, 269.

(11) Talik, P.; Zabkowskawaclawek, M.; Waclawek, W. J. Mater. Sci. 1992, 27, 6807.

(12) George, V.; Jenkins, T. F.; Leggett, D. C.; Cragin, J. H.; Phelan, J. M.; Oxley, J. C.; Pennington, J. Proc. SPIE 1999, 3710, 258.

(13) Cummings, C., private communication.

(14) Patel, S. V.; Jenkins, M. W.; Hughes, R. C.; Yelton, W. G.; Ricco, A. J. Anal. Chem. 2000, 72,1532.

(15) Park, J.; Groves, W. A.; Zellers, E. T. Anal. Chem; 1999, 71, 3877. 